\title{
| COMMENTAIRE DU CNRS
}

\author{
Philippe Bompard
}

Tout d'abord il faut signaler l'importance du travail d'équipe réalisé par les deux Académies (lié au dynamisme et à la capacité de mobilisation de ses auteurs) et l'intérêt du document, en particulier pour tout métallurgiste qui y reconnaîtra les nombreuses et difficiles évolutions du contexte de cette discipline. Les recommandations 1 à 5 sont en particulier tout à fait pertinentes.

La tonalité générale de la synthèse du rapport présente un caractère assez " défensif », qui est sans doute volontaire. Conjuguée à une certaine absence de « ruptures technologiques fortes » observables en métallurgie (notion de progrès continu), cette tonalité défensive pourrait faire perdre une partie de l'impact recherché auprès des décideurs politiques. En effet ceux-ci sont amenés à arbitrer avec $d^{\prime}$ autres disciplines comme l'informatique, l'électronique, les sciences du vivant, ou même d'autres parties très actives de la science des matériaux (composites, matériaux à propriétés électroniques et optiques, approches nano...). II serait peut-être souhaitable que cette synthèse inscrive la métallurgie dans une dynamique plus positive et mieux liée aux évolutions et priorités de la nation.

Le rapport considère que la part de la recherche publique en France est insuffisante, en particulier en métallurgie. Cependant c'est surtout la part de la recherche privée dans ce domaine qui est insuffisante, conséquence inéluctable de la désindustrialisation de la France dans ce domaine, et du départ progressif des centres de recherche et des centres de décision vers d'autres pays. C'est donc tout d'abord à travers une politique de réindustrialisation que les effets négatifs sur la recherche et les laboratoires de métallurgie, bien décrits dans le rapport, seront le mieux combattus.

Les priorités du CNRS, telles qu'elles sont affichées dans son Contrat Objectifs Moyens, s'inscrivent au cœur des priorités sociétales de la nation (vivant, environnement, énergie, information...). II ne s'agit pas d'effets de mode, mais de priorités à long terme. Le CNRS ne considère donc pas la métallurgie (ni beaucoup d'autres disciplines) comme une priorité en soi, et doit faire une place légitime à des disciplines en émergence comme les sciences de l'information. Cependant, bien conscient de la place transversale qu'occupe la science des matériaux, le CNRS fait dans ce domaine un effort important en soutenant, depuis le début des années 1980, un programme interdisciplinaire de recherche (PIR) sur les matériaux, en pratique souvent métalliques. Ce programme est doté 
d'un budget conséquent, et est piloté au niveau de l'ensemble des Instituts. Le comité de pilotage définit les axes de recherche du programme en s'appuyant fortement sur la demande et le partenariat des grandes entreprises et/ou des EPIC du domaine. Par ailleurs, on notera également que les programmes ANR, blanc ou thématiques, disposent d'un budget conséquent, et font également une part importante à la science des matériaux, et à la métallurgie.

Au CNRS, la métallurgie "classique » relève, comme l'indique le rapport, de plusieurs sections du Comité national : principalement la 15 (chimie), mais également la 05 (physique) la 09 (mécanique) et la 10 (procédés). Le rapport propose que l'ensemble des métallurgistes soient rassemblés dans une même section, pour favoriser les échanges entre eux. Cette idée peut être examinée, mais ses inconvénients potentiels sont importants : risque de formation d'un " pré carré » défensif, risque de domination à terme d'une seule " approche » de la métallurgie, risque de recrutement au profit d'une seule famille de laboratoires. Une autre possibilité pourrait être la création d'une section interdisciplinaire (CID) ad hoc, composée de membres déjà en exercice dans d'autres sections (comme c'est le cas pour la bio-informatique par exemple). Mais le nombre de postes ouverts au concours dans une CID est nécessairement limité, et l'effet de rejet des candidats "métallurgistes » par les sections classiques vers cette CID deviendrait alors un risque supplémentaire important.

Le rapport reproche à la recherche publique en métallurgie d'être loin de I'industrie. Cependant, les laboratoires associés au CNRS, relevant de la métallurgie (Insis et INSC essentiellement), ont une part largement majoritaire de leur budget de recherche (hors salaires) constituée de contrats de recherche partenariale bilatéraux avec des entreprises, et/ou de projets ANR obtenus sur des programmes thématiques ciblés, en partenariat avec des industriels (en moyenne de 60 à $70 \%$ ). Cette recherche publique est donc proche des préoccupations des entreprises et ce sont leurs problèmes concrets dont elle s'inspire pour sa recherche amont. C'est le " comprendre pour faire », motivé par les défis technologiques autant que par les enjeux de société. Mais là encore la métallurgie est trop peu visible de ces deux points de vue, en particulier dans les médias généraux et scientifiques, et donc peu visible des jeunes étudiants, des futurs thésards, et donc futurs chercheurs... Ce déficit d'image n'est pas un effet de mode, il est lié aux causes citées antérieurement et devra être pris en compte et corrigé sur la durée, aussi bien par les entreprises que par les laboratoires.

Une conséquence importante de la baisse de la demande industrielle de recherche partenariale en métallurgie, et de son manque de suivi, est la diminution du nombre de laboratoires et de chercheurs publics qui s'y consacrent, et leur concentration sur quelques laboratoires, en général historiques, du domaine. Cependant, même s'ils ont des difficultés, pour les raisons évoquées précédemment, à recruter des étudiants, ces laboratoires sont toujours d'excellente qualité, 
et sont toujours soutenus par le CNRS (postes, bourses, crédits incitatifs sur programme). Produisant de nombreux jeunes docteurs métallurgistes en partenariat avec l'industrie (Cifre, contrats de recherche), ces laboratoires pourront servir, le moment venu, de germes à un redémarrage quantitatif de cette discipline. Il faut remarquer que la capacité nouvelle et croissante de la science des matériaux à modéliser numériquement et prédire quantitativement les microstructures et les propriétés par des approches multi-échelles très complexes, ouvre à cette discipline de grandes perspectives. C'est peut-être une révolution comparable à celle connue par la mécanique au début des années 1980, avec le calcul de structure par éléments finis, et ses évolutions vers la CAO et la conception intégrée.

Malgré le nombre d'emplois à pourvoir évalué par le rapport, les étudiants, mais aussi les établissements d'enseignement, co-tutelles de nos laboratoires CNRS, perçoivent une faiblesse des offres d'emplois de haut niveau en métallurgie et une diminution de la demande de recherche dans ce domaine. Le renouvellement des postes de chercheurs et $d^{\prime}$ IT au sein des laboratoires se trouve ainsi fréquemment en situation d'arbitrage défavorable avec des disciplines de l'ingénieur plus récentes et dynamiques comme les STIC, ou comme le génie industriel plus proche. Mais si les écoles d'ingénieurs en particulier, et leurs enseignants, qui ont de nombreux partenariats et chaires avec les entreprises, recevaient de la part des industriels métallurgistes un message significatif $\mathrm{d}^{\prime} \mathrm{em}$ bauche et de partenariat de recherche sur la durée, il serait alors plus simple de préserver les formations, les postes et les laboratoires. On ne peut que constater un écart de perception des besoins entre responsables techniques des entreprises (pris en compte dans le rapport) et les responsables $\mathrm{RH}$ de ces mêmes entreprises. Cet écart devra être réduit, pour que la métallurgie regagne en intérêt et en audience auprès des étudiants et des établissements, au bénéfice de nos laboratoires. 


\section{KVSDJHIQQMQWRQDCDOHWEDQN}

